\title{
Finite Time Control of Robotic Manipulators with Position Output Feedback
}

\author{
Ali Abooee, Masoud Moravej Khorasani, and Mohammad Haeri \\ -Advanced Control Systems Lab, Sharif University of Technology, Tehran, Iran
}

\begin{abstract}
This paper deals with the robust finite time tracking of desired trajectories for a wide group of robotic manipulators in spite of unknown disturbances, uncertainties, and saturations of actuators while only manipulator's positions are available and its velocities are not measurable physically. A new form of chatteringfree second order fast nonsingular terminal sliding mode control scheme is introduced to design input torques for fulfilling the determined tracking objective in the adjustable total finite settling time. The proposed control algorithm is incorporated with two nonlinear observers to estimate disturbances and velocities of joints within finite settling times. The global finite time stability of the closed-loop manipulator is analytically proved. Finally, a numerical simulation is carried out to verify the effectiveness of the designed input torques.
\end{abstract}

Keywords. Finite settling time; Finite time disturbance observer; Finite time velocity observer; Second order fast nonsingular terminal sliding mode control; Terminal sliding surface.

\section{Introduction}

Recently, referring to widespread exploitation of robotic manipulators in industrial

This is the author manuscript accepted for publication and has undergone full peer review but has not been through the copyediting, typesetting, pagination and proofreading process, which may lead to differences between this version and the Version of Record. Please cite this article as doi: $10.1002 /$ rnc.3721

This article is protected by copyright. All rights reserved. 
applications [1], desired trajectory tracking problem with high precision and fast convergence has become a hot topic in the control community. In consequence, many investigations have been done on this issue [2-11]. The incipient control strategies for tackling the tracking problem, particularly computed torque control and inversedynamics control [2], have two basic shortcomings. First, they can only guarantee the globally asymptotic stability with infinite settling time [2-6]. Second, these primary control methods are not robust against manipulator's uncertainties, external disturbances, and un-modeled friction forces [5].

To overcome the first deficiency, finite time control approaches have been introduced using the finite time stability concept $[7,8]$ where speed up the convergence rate along with high precision and as a result, finite time trajectory tracking is achieved for manipulators $[9,10]$. To cope with the second weakness, researchers have employed the sliding mode control (SMC) technique because of its main feature, namely, robustness against uncertainties, parameter variations, and disturbances [2-4, 6]. Since SMC applies discontinuous control laws, the undesirable chattering phenomenon are seen in the control efforts. Moreover, linear sliding surfaces are used in SMC and, in consequence, only asymptotically stability is provided for tracking problem [6]. By incorporating the conventional SMC and the finite time stability concept, a new terminal SMC (TSMC) scheme [7, 8] has been proposed that accomplishes both the trajectory tracking in a finite settling time [1, 11-15] and robustness against uncertainties $[15,16]$.

Three major drawbacks of a TSMC are occurrence of singularity, high sensitivity of the convergence rate to initial conditions, and chattering phenomenon [11-15]. The 
singularity occurs due to existence of nonlinear terms with fractional negative powers in sliding surfaces and control laws. This unwanted problem causes in large control efforts when tracking errors reach the neighborhood of zero [11-16]. Researchers have eliminated the singularity problem by introducing nonsingular TSMC (NTSMC) technique for rigid manipulators but other two mentioned problems still exist in the suggested NTSMC [1, 17-19]. By combining the second order sliding method [20-22] and NTSMC technique, the second order NTSMC scheme has been proposed to ensure the finite time trajectory tracking for robotic manipulators [23-25]. This strategy removes the chattering occurrence with its continuous control laws $[21,22]$ and possesses all advantages of NTSMC approach as well.

Literature survey on finite time control of manipulators reveals that most of the proposed methods require position and velocity measurements of manipulator's joints [11-15, 17-19, 23-25]. Moreover, some of these methods utilize direct measurements of accelerations or their estimations as well [23]. In fact, these methods have presumed that all state variables are measurable or available which is not true for many practical manipulators in general. While the joints position can be measured accurately by using precise encoders the velocity measurements need additional sensors such as tachometers that increase cost, weight, and size of servo-drive systems. Also, measurements obtained using tachometers are easily contaminated by noise [25].

Alternatively, some researchers have employed velocity observers and incorporated the observers with the finite time trajectory tracking schemes [23, 26-38]. This approach has two major defects. First, most of the observers provide asymptotic 
convergence of estimation errors and, in consequence, the finite time stability of the closed-loop system is destroyed [27-29]. Second, control laws and velocity observers are designed individually and, thus, since the separation principle does not hold for nonlinear systems in general the finite time stability of the closed-loop manipulator should be assured again [23, 30, 32].

In practical realizations of input torques, saturation of actuators, which are caused due to physical limitations and safety reasons, degrades the closed-loop system's performance. Therefore, the input constraints should be considered along with the analytical studies related to design of the control laws. Only a few researches have investigated this issue $[33,34]$.

In this paper a modified second order fast NTSMC (FNTSMC) is incorporated with finite time nonlinear disturbance observer and full order velocity observer to achieve the finite time tracking for robotic manipulators only by applying position feedback. The manipulator is subjected to uncertainties, unbounded disturbances, and actuators' saturation. Note that the measurement of acceleration is not required in the proposed control scheme. When compared to other works, the major contributions of this paper are listed as follows.

- Introducing a control scheme which is applicable for finite time stabilization and tracking of a wide group of nonlinear systems.

- Applying only output feedback of positions and estimated velocities.

- Establishing an adjustable total finite settling time for converging of tracking errors to zero.

- Reconstruction of unknown lumped uncertainties and disturbances in a finite 
settling time by proposing a nonlinear observer.

- Estimation of manipulator's velocities in a finite settling time by designing a full order nonlinear observer.

- Tackling the practical problems of actuators such as saturation and dead-zone functions.

- Introducing a new form of terminal sliding surfaces which guarantees finite time convergence of the system to sliding motion.

- Eliminating singularity problem due to using some arbitrary positive fractional powers in the proposed control laws.

- Reduction of high frequency switching of control inputs by utilizing the second order sliding mode approach.

- Decreasing the total finite settling time and reduction of control energy by proper adjustment of arbitrary coefficients used in the suggested control scheme.

The outline of the paper is provided as follows. Section 2 reviews some mathematical lemmas and includes the robotic manipulator's description along with problem formulation of globally finite time tracking. Section 3 is devoted to design of finite time disturbance observer and input torques considering fully measurable closed-loop manipulator system. Also, globally finite time stability of the closed-loop system is proved. A full order finite time velocity observer by using output feedback of positions is designed in Section 4. By applying this observer, the suggested input torques are modified and the proof of finite time stability is investigated again. Section 5 describes a numerical example to verify the theoretical results. Section 6 gives conclusions of the paper. 
Notations. $C^{2}[0 \infty)$ denotes the space of all functions with domain $[0 \infty)$ and bounded and continuous first and second order derivatives. $\operatorname{sgn}(x)$ is the signum function of real number $x$. For a typical vector $x \in \mathbb{R}^{n},\|x\|$ is the 2-norm of $x$ that is $\|x\|=$ $\sqrt{x^{T} x}$. Also, for a non-square matrix $A \in \mathbb{R}^{n \times m},\|A\|$ is the induced matrix 2-norm defined by $\|A\|=\sqrt{\lambda_{\max }\left(A^{T} A\right)}$ where $\lambda_{\max }\left(A^{T} A\right)$ is the maximum eigenvalue of $A^{T} A \in \mathbb{R}^{m \times m}$. By considering $x=\left[\begin{array}{lll}x_{1} & \ldots & x_{n}\end{array}\right]^{T},|x| \in \mathbb{R}^{n}$ and $\operatorname{sgn}(x) \in \mathbb{R}^{n}$ are defined as $|x|=\left[\begin{array}{lll}\left|x_{1}\right| & \ldots & \left|x_{n}\right|\end{array}\right]^{T}$ and $\operatorname{sgn}(x)=\left[\operatorname{sgn}\left(x_{1}\right) \ldots \operatorname{sgn}\left(x_{n}\right)\right]^{T}$. For $x=\left[\begin{array}{lll}x_{1} & \cdots & x_{n}\end{array}\right]^{T}$, $y=\left[\begin{array}{lll}y_{1} & \cdots & y_{n}\end{array}\right]^{T}$, and a real number $\vartheta \in \mathbb{R},|y| \operatorname{sgn}(x) \in \mathbb{R}^{n}, \operatorname{sig}^{\vartheta}(x) \in \mathbb{R}^{n}, y \operatorname{sig}^{\vartheta}(x) \in$ $\mathbb{R}^{n}$, and $\operatorname{sig}^{y}(x) \in \mathbb{R}^{n}$ define $|y| \operatorname{sgn}(x)=\left[\left|y_{1}\right| \operatorname{sgn}\left(x_{1}\right) \cdots\left|y_{n}\right| \operatorname{sgn}\left(x_{n}\right)\right]^{T}, \operatorname{sig}^{\vartheta}(x)=$ $\left[\left|x_{1}\right|^{\vartheta} \operatorname{sgn}\left(x_{1}\right) \cdots\left|x_{n}\right|^{\vartheta} \operatorname{sgn}\left(x_{n}\right)\right]^{T}, y \operatorname{sig}^{\vartheta}(x)=\left[y_{1}\left|x_{1}\right|^{\vartheta} \operatorname{sgn}\left(x_{1}\right) \cdots y_{n}\left|x_{n}\right|^{\vartheta} \operatorname{sgn}\left(x_{n}\right)\right]^{T}$, and $\operatorname{sig}^{y}(x)=\left[\left|x_{1}\right|^{y_{1}} \operatorname{sgn}\left(x_{1}\right) \quad \ldots \quad\left|x_{n}\right|^{y_{n}} \operatorname{sgn}\left(x_{n}\right)\right]^{T}$, respectively.

\section{Mathematical Tools, System Description, and Problem Statement}

Three lemmas are reviewed which will be utilized in the following sections. The main definition of globally finite time stability has been given in $[7,8]$.

Lemma 1 [17]. Consider nonlinear system $\dot{x}(t)=f(x), x \in \mathbb{R}^{n}, x(0)=x_{0}$ with the equilibrium point $x=0$. Assume that there exist three real numbers $\rho_{1}>0, \rho_{2}>0$, $0<\beta<1$, and a continuously differentiable positive function $V(x): \mathbb{R}^{n} \rightarrow \mathbb{R}$ such that the inequality $\dot{V}(x)+\rho_{1} V(x)+\rho_{2} V^{\beta}(x) \leq 0$ is always fulfilled for any solution $x\left(t, x_{0}\right)$ of the mentioned system. Then, the equilibrium point $x=0$ is globally finite time stable and the finite convergence time $T_{S}$, which is called finite settling time, satisfies inequality $T_{s}\left(x_{0}\right) \leq\left(\rho_{1}(1-\beta)\right)^{-1}\left(\ln \left(\rho_{1} V^{1-\beta}\left(x_{0}\right)+\rho_{2}\right)-\ln \rho_{2}\right)$. 
Lemma 2 [37, 38]. For any real numbers $a_{i}$ and $0<\beta<1$ inequalities (i): $\sum_{i=1}^{n}\left|a_{i}\right|^{\beta} \geq\left(\sum_{i=1}^{n}\left|a_{i}\right|\right)^{\beta}$ and $(i i): \sum_{i=1}^{n}\left|a_{i}\right|^{1+\beta} \geq \sqrt{\left(\sum_{i=1}^{n}\left|a_{i}\right|^{2}\right)^{1+\beta}}$ are always fulfilled.

Lemma 3 [7]. Consider a double integrator system which is expressed by differential equations $\dot{x}=v, \dot{v}=u$ where $u$ is the control input and $x$ and $v$ are the state variables. This system is globally finite time stable by implementing control input (1). $u=-|v|^{\chi} \operatorname{sgn}(v)-|\phi(x, v)|^{\chi(2-\chi)^{-1}} \operatorname{sgn}(\phi(x, v))$, $\phi(x, v)=x+(2-\chi)^{-1}|v|^{2-\chi} \operatorname{sgn}(v)$,

where tuning coefficient $0<\chi<1$ can be used to adjust the finite convergence time. Furthermore, by using control input (1), state variables $x$ and $v$ exactly converge to zero in the finite convergence time specified as

$T(x(0), v(0)) \leq(c(1-\chi))^{-1}(3-\chi)(V(x(0), v(0)))^{\frac{1-\chi}{3-\chi}}$

where positive function $V(x, v)$ and coefficient $c$ are defined such that arbitrary parameters $\mu$ and $\Upsilon$ fulfill constraints $0<\mu<1$ and $\Upsilon>1$ respectively [7].

$V=\frac{2-\chi}{3-\chi}|\phi(x, v)|^{\frac{3-\chi}{2-\chi}}+\mu v \phi(x, v)+\frac{r}{3-\chi}|v|^{3-\chi}$ $c=-\max _{(x, v) \in \psi} \dot{V}(x, v)$ with $\psi=\{(x, v): V(x, v)=1\}$

A large group of $n$-link rigid robotic manipulators could be described by differential equation (4) where $n$ describes the number of degrees of freedom [23].

$M(x) \dot{v}+H(x, v)+G(x)+D(t, x, v)=\tau$

$x \in \mathbb{R}^{n}, v \in \mathbb{R}^{n}$ and $\dot{v} \in \mathbb{R}^{n}$ represent positions, velocities, and accelerations of manipulator's joints. The symmetric positive definite matrix $M(x) \in \mathbb{R}^{n \times n}$ denotes the inertia. $H(x, v) \in \mathbb{R}^{n}$ is defined by $H=B(x)\langle v, v\rangle+C(x) v^{2}$ such that $B(x) \in$ 
$\mathbb{R}^{n \times 0.5 n(n-1)}$ is Coriolis's coefficients and $C(x) \in \mathbb{R}^{n \times n}$ is centrifugal forces. $\langle v, v\rangle \in$ $\mathbb{R}^{n \times 0.5 n(n-1)} \quad$ and $\quad v^{2} \in \mathbb{R}^{n}$ imply $\langle v, v\rangle=\left[\begin{array}{llllll}v_{1} v_{2} & \cdots & v_{1} v_{n} & \cdots & v_{n-1} v_{n}\end{array}\right]^{T}$ and $v^{2}=$ $\left[\begin{array}{lll}v_{1}^{2} & \cdots & v_{n}^{2}\end{array}\right]^{T} . G(x) \in \mathbb{R}^{n}$ and $\tau \in \mathbb{R}^{n}$ indicate gravitational forces and torques applied to the manipulator's joints, respectively [23]. The lumped vector $D(t, x, v) \in \mathbb{R}^{n}$, which is assumed to be absolutely continuous, stands for external disturbances, uncertainties, and unmodeled dynamics. Also, $\dot{D}(t, x, v) \in \mathbb{R}^{n}$ is considered to be locally bounded Lebesgue measurable to guarantee the existence of control law $\tau$.

Assumption 1. $D$ satisfies inequalities $\|D\| \leq \alpha_{0}(t)$ and $\|\dot{D}\| \leq \alpha_{1}(t)$ where $\alpha_{0}(t)$ and $\alpha_{1}(t)$ are known positive functions.

Due to practical limitations or safety reasons, the electrical motors of joints should not supply the designed input torques completely. Therefore, in this study, saturation constraint is considered for joints' actuators.

Assumption 2 [15, 34]. Based on the constraints, it is assumed that non-symmetric saturation functions of designed torques $\tau_{j}, j=1, \ldots, n$ are applied to the manipulators. The saturation functions describing the actuator's constraints are considered as follows.

$u_{j}=\left\{\begin{array}{ccc}u_{\max _{j}}, & \text { if } & \tau_{j}>u_{\max _{j}} \\ \tau_{j}, & \text { if } & -u_{\min _{j}} \leq \tau_{j} \leq u_{\max _{j}}, j=1, \ldots, n . \\ -u_{\min _{j}}, & \text { if } & \tau_{j}<-u_{\min _{j}}\end{array}\right.$

$u_{j}$ is output of the $j^{\text {th }}$ actuator and $u_{\max _{j}}$ and $u_{\min _{j}}$ are its known maximum and minimum values.

By defining the state variables as $x=\left[\begin{array}{lll}x_{1} & \cdots & x_{n}\end{array}\right]^{T}$ and $v=\left[\begin{array}{lll}v_{1} & \cdots & v_{n}\end{array}\right]^{T}$ where $x$ and $v$ represent positions and velocities respectively, the state space model of the robotic 
manipulator in (4) is derived as follows

$\dot{x}=v$

$\dot{v}=f(x, v)+N(t, x, v)+g(x)(\tau+\Delta u)$

where $g(x)=M^{-1}(x), f=-g(x) H(x, v)-g(x) G(x)$, and $N=-g(x) D(t, x, v)$.

The known term $\Delta u$ is calculated as $\Delta u=u-\tau$ where $u$ is the vector of outputs of saturation functions (5). Based on Assumption 1 and inequality $\left\|M^{-1}\right\| \leq \lambda_{\max }\left(M^{-1}\right)$ [23], it is found that $\|N\| \leq \alpha_{0}(t) \lambda_{\max }\left(M^{-1}\right)$ where $\lambda_{\max }\left(M^{-1}\right)$ is the largest eigenvalue of matrix $M^{-1}$. Notice that (6) describes a fully actuated system.

Preposition 1. According to definition $N=-g(x) D(t, x, v)$, the inequality $\|\dot{N}\| \leq$ $\alpha_{2}(t)$ is always satisfied where $\alpha_{2}(t)$ is given by $\alpha_{2}(t)=\alpha_{0}(t)\|\dot{g}(x)\|+$ $\alpha_{1}(t) \lambda_{\max }\left(M^{-1}\right)$.

The proof of this preposition is simple and is omitted.

Assumption 3 [35]. According to the work space of physical robotic manipulators, it is assumed that $v$ fulfills $\|v\| \leq \kappa$ where the upper bound $\kappa$ is a known constant.

Since $v$ includes velocities of joints, this assumption is reasonable and acceptable for industrial robot manipulators due to practical restrictions and safety reasons. Note that the maximum velocity of each joint is usually given in the data sheet.

Assumption 4. $x$ as an output feedback vector is measurable.

As the control goal, torque $\tau$ should be designed such that all joints of manipulator track desired trajectories in an adjustable finite time in spite of actuator saturation, unmodeled dynamics, uncertainties, and external disturbances. The desired trajectory $x_{d}=\left[\begin{array}{lll}x_{d_{1}} & \cdots & x_{d_{n}}\end{array}\right]^{T}$ is determined by the user and it is assumed that the trajectories 
$x_{d_{j}}, j=1, \ldots, n$ belong to $C^{2}[0 \infty)$. Also, $v_{d}=\left[v_{d_{1}} \cdots v_{d_{n}}\right]^{T}$ denotes the derivative of desired trajectory. At first, it is assumed that $x$ and $v$ are directly measurable. A finite time disturbance observer $\widehat{D}$ is designed to estimate $D$ within a finite time. Then, by applying disturbance observer $\widehat{D}$ and measured $x$ and $v$, a novel chatteringfree second order FNTSMC scheme is developed to determine $\tau$ for fulfilling the finite time tracking in finite settling time $T_{s_{t}}=T_{S_{r}}+T_{S_{S}}$ where settling times $T_{S_{r}}$ and $T_{S_{S}}$ will be introduced later. By defining tracking errors as $e_{x}=\left[\begin{array}{lll}e_{x_{1}} & \cdots & e_{x_{n}}\end{array}\right]^{T}=x-$ $x_{d}$ and $e_{v}=\left[\begin{array}{lll}e_{v_{1}} & \cdots & e_{v_{n}}\end{array}\right]^{T}=v-v_{d}$, the tracking error dynamics of manipulator (4) are obtained as below.

$\dot{e}_{x}=e_{v}$

$\dot{e}_{v}=f(x, v)+N(t, x, v)+g(x)(\tau+\Delta u)-\dot{v}_{d}$

To achieve the described control aim, input torque $\tau$ is designed for globally finite time stabilizing of dynamic system (7).

Since the direct measurement of joints' velocities is an expensive process in practice and the measured data are always noisy, it is usually preferred to estimate $v$. Here, a finite time nonlinear observer is designed to estimate $v$ in an adjustable settling time $T_{S_{o}}$. Then, in the designed control law $\tau, v$ is replaced by its estimation $\hat{v}$. In consequence, by considering the modified input torque, the global finite time stability of closed-loop system (7) is studied and investigated again.

\section{Design of the Disturbance Observer and Input Torques}

In the sequel, a finite time disturbance observer is designed to estimate $D$. 
$\widehat{N}=-K \sigma-f(x, v)-\varepsilon \operatorname{sig}^{\vartheta}(\sigma)-\alpha_{0}(t) \lambda_{\max }\left(M^{-1}\right) \operatorname{sgn}(\sigma)-|f(x, v)| \operatorname{sgn}(\sigma)$

$\dot{z}=-K \sigma+g \tau+g \Delta u-\dot{v}_{d}-\alpha_{0}(t) \lambda_{\max }\left(M^{-1}\right) \operatorname{sgn}(\sigma)-\varepsilon \operatorname{sig}^{\vartheta}(\sigma)-|f(x, v)| \operatorname{sgn}(\sigma)$

$\sigma=z-e_{v}, \widehat{D}=g^{-1} \widehat{N}$,

In this observer, $\sigma=\left[\begin{array}{lll}\sigma_{1} & \cdots & \sigma_{n}\end{array}\right]^{T}$ is a subsidiary vector and $\widehat{D}$ is the estimation of $D$ which converges to it in a finite time. $K \sigma \in \mathbb{R}^{n}$ and $\varepsilon \in \mathbb{R}^{n}$ are defined as $K \sigma=$ $\left[\begin{array}{lll}k_{1} \sigma_{1} & \cdots & k_{n} \sigma_{n}\end{array}\right]^{T}$ and $\varepsilon=\left[\begin{array}{lll}\varepsilon_{1} & \cdots & \varepsilon_{n}\end{array}\right]^{T}$ where, $k_{j}>0, \varepsilon_{j}>0$, and $0<\vartheta<1$ are arbitrary coefficients which should be determined by the designer.

In the following discussion, it is proven that the estimation errors related to the disturbance observer converged to zero in a finite time.

By developing the second order FNTSMC method, the vector of torques $\tau$ is designed to finite time stabilize dynamic system (7) in total settling time $T_{s_{t}}$. Note that for designing $\tau$, the output of suggested disturbance observer (8) $(\widehat{N})$ is employed.

The second order terminal sliding surface $s=\left[\begin{array}{lll}s_{1} & \cdots & s_{n}\end{array}\right]^{T}$ is proposed as follows.

$s=\sigma+\dot{e}_{v}+\operatorname{sig}^{\chi x}\left(e_{v}\right)+\operatorname{sig}^{\chi_{v}}\left(\phi\left(e_{x}, e_{v}\right)\right)$

where $\chi_{x} \in \mathbb{R}^{n}$ and $\chi_{v} \in \mathbb{R}^{n}$ are defined as $\chi_{x}=\left[\begin{array}{lll}\chi_{x_{1}} & \cdots & \chi_{x_{n}}\end{array}\right]^{T}$ and $\chi_{v}=\left[\begin{array}{lll}\chi_{v_{1}} & \cdots & \chi_{v_{n}}\end{array}\right]^{T}$ where $\chi_{x_{j}} \in(0,1)$ are arbitrary parameters and $\chi_{v_{j}}=\chi_{x_{j}}\left(2-\chi_{x_{j}}\right)^{-1}$ for $j=1, \ldots, n$. $\phi\left(e_{x}, e_{v}\right) \in \mathbb{R}^{n} \quad$ is defined as $\phi\left(e_{x}, e_{v}\right)=\left[\phi_{1}\left(e_{x_{1}}, e_{v_{1}}\right) \cdots \phi_{n}\left(e_{x_{n}}, e_{v_{n}}\right)\right]^{T}$ where $\phi_{j}\left(e_{x_{j}}, e_{v_{j}}\right)$ is given by $\phi_{j}\left(e_{x_{j}}, e_{v_{j}}\right)=e_{x_{j}}+\left(2-\chi_{x_{j}}\right)^{-1}\left|e_{v_{j}}\right|^{2-\chi_{x_{j}}} \operatorname{sgn}\left(e_{v_{j}}\right)$ for $j=1, \ldots, n$.

The control law $\tau$ is suggested as follow.

$\tau=g^{-1}\left(-f(x, v)-\operatorname{sig} \chi_{x}\left(e_{v}\right)-\operatorname{sig} \chi_{v}\left(\phi\left(e_{x}, e_{v}\right)\right)+\dot{v}_{d}+\tau_{r}\right)-\Delta u$

$\dot{\tau}_{r}=-\omega s-\Omega \operatorname{sig}^{\vartheta}(s)-\widehat{N}-\left(\alpha_{0}(t) \lambda_{\max }\left(M^{-1}\right)+\alpha_{2}(t)\right) \operatorname{sgn}(s)$

$\omega s \in \mathbb{R}^{n}$ and $\Omega \in \mathbb{R}^{n}$ are defined as $\omega s=\left[\begin{array}{lll}\omega_{1} s_{1} & \cdots & \omega_{n} s_{n}\end{array}\right]^{T}$ and $\Omega=\left[\begin{array}{lll}\Omega_{1} & \cdots & \Omega_{n}\end{array}\right]^{T}$ respectively where $\omega_{j}>0$ and $\Omega_{j}>0$ are arbitrary constants that are selected by 
designer.

Theorem 1 proves the existence of terminal sliding motion $s=0$ by applying the proposed control in (10) to system (7). In other words, tracking errors presented by system (7) reaches sliding mode $s=0$ in finite time $T_{s_{r}}$. Moreover, it is concluded that $\sigma=0$ is fulfilled for $t \geq T_{s_{r}}$. Theorem 2 demonstrates that the estimation errors of disturbances reach zero in finite time $T_{s_{r}}$. Furthermore, Theorem 3 shows that sliding motion $s=0$ is globally finite time stable that means the tracking errors, which had been converged to $s=0$, reach zero in finite time $T_{S_{s}}$.

Theorem 1. Consider sliding surface (9) and input torque (10). If the control law in (10) is applied to manipulator (4), then the tracking errors, presented by system (7), will reach terminal sliding motion $s=0$ in adjustable finite time $T_{s_{r}}$. Also, $\sigma=0$ is satisfied for $t>T_{s_{r}}$. The upper bound for $T_{s_{r}}$ is given by

$T_{s_{r}} \leq(\eta(1-\vartheta))^{-1}\left(\ln \left(\eta \sqrt{\left(\sum_{j=1}^{n} s_{j}^{2}(0)+\sigma_{j}^{2}(0)\right)^{1-\vartheta}}+\gamma\right)-\ln \gamma\right)$

where $\eta$ and $\gamma$ are defined as $\eta=\min \left(\min \omega_{j}, \min k_{j}\right)$ and $\gamma=\min \left(\min \varepsilon_{j}, \min \Omega_{j}\right)$.

Proof. The candidate Lyapunov function is considered as $V=0.5\left(s^{T} s+\sigma^{T} \sigma\right)$. By placing $\tau$ and $\dot{e}_{v}$ from (10) and (7) into (9), sliding surface $s$ is obtained as $s=N+$ $\tau_{r}+\sigma$ and, in consequence, $\dot{s}=\dot{N}+\dot{\tau}_{r}+\dot{\sigma}$. According to $\dot{\tau}_{r}$ and $\sigma$ given by (10) and (8), $\dot{s}$ is derived as follows.

$\dot{s}=\dot{N}-N-\omega s-\Omega \operatorname{sig}^{\vartheta}(s)-\left(\alpha_{0}(t) \lambda_{\max }\left(M^{-1}\right)+\alpha_{2}(t)\right) \operatorname{sgn}(s)$

The following relation is obtained by applying Cauchy-Schwartz inequalities $s^{T} \dot{N} \leq$ $\|s\|\|\dot{N}\|$ and $-s^{T} N \leq\|s\|\|N\|$, and inequality $\|s\| \leq \sum_{j=1}^{n}\left|s_{j}\right|$. 
$s^{T} \dot{s} \leq\|s\|\|\dot{N}\|+\|s\|\|N\|-\left(\alpha_{0}(t) \lambda_{\max }\left(M^{-1}\right)+\alpha_{2}(t)\right)\|s\|-\sum_{j=1}^{n} \omega_{j} s_{j}^{2}-\sum_{j=1}^{n} \Omega_{j}\left|s_{j}\right|^{\vartheta+1}$

By considering $\|N\| \leq \alpha_{0}(t) \lambda_{\max }\left(M^{-1}\right)$ and Preposition 1 , the following inequality is concluded where $\omega_{\min }=\min \omega_{j}$ and $\Omega_{\min }=\min \Omega_{j}$.

$s^{T} \dot{S} \leq-\omega_{\min } \sum_{j=1}^{n} s_{j}^{2}-\Omega_{\min } \sum_{j=1}^{n}\left|s_{j}\right|^{\vartheta+1}$.

From $(8), \dot{\sigma}$ is determined as

$\dot{\sigma}=-K \sigma-\varepsilon \operatorname{sig}^{\vartheta}(\sigma)-\alpha_{0}(t) \lambda_{\max }\left(M^{-1}\right) \operatorname{sgn}(\sigma)-f(x, v)-|f(x, v)| \operatorname{sgn}(\sigma)-N$.

Therefore, $\sigma^{T} \dot{\sigma}$ is calculated as

$\sigma^{T} \dot{\sigma}=-\sigma^{T}(K \sigma)-\sigma^{T}\left(\varepsilon \operatorname{sig}^{\vartheta}(\sigma)\right)-\alpha_{0}(t) \lambda_{\max }\left(M^{-1}\right)\left(\sum_{j=1}^{n}\left|\sigma_{j}\right|\right)-\sigma^{T} f-|\sigma|^{T}|f|-\sigma^{T} N$

Since $-\sigma^{T} f-|\sigma|^{T}|f|$ is always negative and inequalities $-\sigma^{T} N \leq\|\sigma\|\|N\|$ and $\|\sigma\| \leq \sum_{j=1}^{n}\left|\sigma_{j}\right|$ always hold, (16) is simplified to

$\sigma^{T} \dot{\sigma} \leq-\sum_{j=1}^{n} k_{j} \sigma_{j}^{2}-\sum_{j=1}^{n} \varepsilon_{j}\left|\sigma_{j}\right|^{\vartheta+1}+\|\sigma\|\left(\|N\|-\alpha_{0}(t) \lambda_{\max }\left(M^{-1}\right)\right)$.

By applying $\|N\| \leq \alpha_{0}(t) \lambda_{\max }\left(M^{-1}\right)$ and considering definitions $k_{\min }=\min k_{j}$ and $\varepsilon_{\min }=\min \varepsilon_{j}$, the following inequality is derived.

$\sigma^{T} \dot{\sigma} \leq-k_{\min } \sum_{j=1}^{n} \sigma_{j}^{2}-\varepsilon_{\min } \sum_{j=1}^{n}\left|\sigma_{j}\right|^{\vartheta+1}$

By using inequalities (14) and (18) and definitions $\eta=\min \left(\omega_{\min }, k_{\min }\right)$ and $\gamma=\min \left(\varepsilon_{\min }, \Omega_{\min }\right)$, the derivative of $V=0.5\left(s^{T} s+\sigma^{T} \sigma\right)$ is calculated as

$\dot{V}=s^{T} \dot{s}+\sigma^{T} \dot{\sigma} \leq-\eta \sum_{j=1}^{n}\left(\sigma_{j}^{2}+s_{j}^{2}\right)-\gamma \sum_{j=1}^{n}\left(\left|\sigma_{j}\right|^{\vartheta+1}+\left|s_{j}\right|^{\vartheta+1}\right)$.

Based on inequality (i) of Lemma 2, the following inequality is obtained.

$\dot{V} \leq-2 \eta V-\gamma\left(\sqrt{\left(\sum_{j=1}^{n}\left|\sigma_{j}\right|^{2}\right)^{\vartheta+1}}+\sqrt{\left(\sum_{j=1}^{n}\left|s_{j}\right|^{2}\right)^{\vartheta+1}}\right)$

According to inequality (ii) of Lemma 2, the following inequality is derived.

$\dot{V} \leq-2 \eta V-\gamma \sqrt{\left(\sum_{j=1}^{n}\left(\sigma_{j}^{2}+s_{j}^{2}\right)\right)^{\vartheta+1}}=-2 \eta V-\gamma \sqrt{2^{\vartheta+1}} \sqrt{V^{\vartheta+1}}$ 
By setting $\rho_{1}=2 \eta, \rho_{2}=\gamma \sqrt{2^{\vartheta+1}}, \beta=0.5(\vartheta+1)$, and using Lemma 1 , it is concluded that equations $s=0$ and $\sigma=0$ are attainable within finite time $T_{s_{r}}$ presented by (11). This completes the proof.

Theorem 2. Consider robotic manipulator (4) and suggested disturbance observer (8). Then, $\widehat{D}$ converges to $D$ after finite time $T_{S_{r}}$ expressed by (11).

Proof. According to Theorem 1, it was concluded that for $t \geq T_{s_{r}}, \sigma=0$ and, in consequence, $\dot{\sigma}=0$ is obtained. By defining $\widetilde{N}$ as $\widetilde{N}=\widehat{N}-N$, equation $\widetilde{N}=\dot{\sigma}$ is derived as

$$
\begin{aligned}
\widetilde{N}= & -K \sigma-f(x, v)-\varepsilon \operatorname{sig}^{\vartheta}(\sigma)-\alpha_{0}(t) \lambda_{\max }\left(M^{-1}\right) \operatorname{sgn}(\sigma)-|f(x, v)| \operatorname{sgn}(\sigma)-N \\
= & -K \sigma-\varepsilon \operatorname{sig}^{\vartheta}(\sigma)-\alpha_{0}(t) \lambda_{\max }\left(M^{-1}\right) \operatorname{sgn}(\sigma)-|f| \operatorname{sgn}(\sigma)-N-\dot{e}_{v}+g \tau \\
& +g \Delta u-\dot{v}_{d}+N=z-\dot{e}_{v}=\dot{\sigma}
\end{aligned}
$$

Based on $\widetilde{N}=\dot{\sigma}$, it is found $\widetilde{N}=0$ that means $\widehat{N}=N$ for $t \geq T_{s_{r}}$. Therefore, after finite settling time $T_{S_{r}}$, relation $\widehat{D}=g^{-1} \widehat{N}$ is converted to $\widehat{D}=g^{-1} N$. Consequently, $\widehat{D}$ converges to $D$ in finite time $T_{s_{r}}$ introduced by (11). This completes the proof.

Theorem 3. Consider terminal sliding surface defined by (9). When system (7) reaches sliding motion $s=0$, then tracking errors $e_{x}$ and $e_{v}$ converge to zero within finite time $T_{S_{S}}$ which satisfies the following inequality

$T_{s_{s}} \leq \max _{j}\left\{\left(c_{j}\left(1-\chi_{x_{j}}\right)\right)^{-1}\left(3-\chi_{x_{j}}\right)\left(V_{j}\left(e_{x_{j}}\left(t=T_{s_{r}}\right), e_{v_{j}}\left(t=T_{s_{r}}\right)\right)\right)^{1-\chi_{x_{j}}}\right.$

where positive functions $V_{j}\left(e_{x_{j}}, e_{v_{j}}\right)$ and compact sets $\psi_{j}$ are defined such that arbitrary parameters $\mu_{j}$ and $\Upsilon_{j}$ fulfill constraints $0<\mu_{j}<1$ and $\Upsilon_{j}>1$ respectively. 
$V_{j}=\frac{2-\chi_{x_{j}}}{3-\chi_{x_{j}}}\left|\phi_{j}\left(e_{x_{j}}, e_{v_{j}}\right)\right|^{\frac{3-\chi x_{j}}{2-\chi x_{j}}}+\mu_{j} e_{v_{j}} \phi_{j}\left(e_{x_{j}}, e_{v_{j}}\right)+\frac{r_{j}}{3-\chi_{x_{j}}}\left|e_{v_{j}}\right|^{3-\chi_{x_{j}}}$

$\psi_{j}=\left\{\left(e_{x_{j}}, e_{v_{j}}\right): V_{j}\left(e_{x_{j}}, e_{v_{j}}\right)=1\right\}$

Also, $c_{j}$ is defined as

$c_{j}=-\max _{\left(e_{x_{j}}, e_{v_{j}}\right) \in \psi_{j}} \dot{V}_{j}\left(e_{x_{j}}, e_{v_{j}}\right)$.

Proof. Considering $s=0, \sigma=0$, and sliding surface (9) for $t \geq T_{s_{r}} \dot{e}_{v}$ becomes as

$\dot{e}_{v}=-\operatorname{sig} \chi_{x}\left(e_{v}\right)-\operatorname{sig} \chi_{v}\left(\phi\left(e_{x}, e_{v}\right)\right)$.

Then for $t \geq T_{s_{r}}$ the error dynamics in (7) would be as follows

$\dot{e}_{x}=e_{v}$

$\dot{e}_{v}=-\operatorname{sig}^{\chi x}\left(e_{v}\right)-\operatorname{sig}^{\chi_{v}}\left(\phi\left(e_{x}, e_{v}\right)\right)$

By expanding (27) for $j=1, \ldots, n$, it can be seen that the sliding motion for system (7) includes $n$ independent double integrator subsystems with the following dynamics.

$\dot{e}_{x_{j}}=e_{v_{j}}$

$\dot{e}_{v_{j}}=-\left|e_{v_{j}}\right|^{\chi_{x_{j}}} \operatorname{sgn}\left(e_{v_{j}}\right)-\left|\phi_{j}\left(e_{x_{j}}, e_{v_{j}}\right)\right|^{\chi_{v_{j}}} \operatorname{sgn}\left(\phi_{j}\left(e_{x_{j}}, e_{v_{j}}\right)\right)$

For each subsystem, candidate Lyapunov function $V_{j}$ and compact set $\psi_{j}$ are defined as (24) [7]. By using the results in [7] and Lemma 3, the following inequality is obtained for all $\left[e_{x_{j}} e_{v_{j}}\right]^{T} \in \mathbb{R}^{2}$.

$\dot{V}_{j}\left(e_{x_{j}}, e_{v_{j}}\right) \leq c_{j}\left(V_{j}\left(e_{x_{j}}, e_{v_{j}}\right)\right)^{\frac{2}{3-\chi x_{j}}}$, where $c_{j}=-\max _{\left[e_{x_{j}} e_{v_{j}}\right]^{T} \in \psi_{j}} \dot{V}_{j}\left(e_{x_{j}}, e_{v_{j}}\right)$

Consequently, tracking errors $e_{x_{j}}$ and $e_{v_{j}}$ for each subsystem converge to zero in finite time ${ }_{j} T_{S_{S}}$ which satisfies the following inequality [7].

$$
{ }_{j} T_{s_{s}} \leq\left(3-\chi_{x_{j}}\right)\left(c_{j}\left(1-\chi_{x_{j}}\right)\right)^{-1}\left(V_{j}\left(e_{x_{j}}\left(T_{s_{r}}\right), e_{v_{j}}\left(T_{s_{r}}\right)\right)\right)^{\frac{1-\chi x_{j}}{3-\chi x_{j}}}
$$

It is clear that for all $n$ independent double integrator subsystems described by (28), 
finite settling time $T_{S_{S}}$, presented by (23), exists such that all tracking errors reach zero for $t \geq T_{s_{r}}+T_{S_{s}}$. Finally, sliding motion $s=0$ of system (7), described by (27), is globally finite time stable. This completes the proof.

Remark 1. Disturbance observer (8) and input torque (10) contain optional positive parameters $\varepsilon_{j}, \vartheta, k_{j}, \Omega_{j}, \omega_{j}$, and $\chi_{x_{j}}$ which could be tuned by designer. A proper selection of these parameters can reduce the control effort and finite settling times $T_{S_{r}}$ and $T_{S_{S}}$ significantly.

Remark 2. Since the second order FNTSMC method is employed to provide the desired tracking, the singularity problem is removed due to applying positive fractional powers $0<\vartheta<1$ and $0<\chi_{x_{j}}<1$. Also, high frequency switching of control signals is reduced greatly.

Remark 3. By considering Theorems 1 and 3, the finite time tracking for manipulator (4) is achieved at $T_{s_{t}}=T_{s_{r}}+T_{s_{s}}$ where $T_{s_{r}}$ and $T_{s_{s}}$ are given in (11) and (23) respectively. Notice that if fractional power $\vartheta$ is selected as 1 , the settling time $T_{s_{r}}$ will become infinity and the finite time tracking becomes an asymptotic one.

Remark 4. When $u_{\max _{j}}$ and $u_{\min _{j}}$ in saturation functions (5) are unknown, $\Delta u$ will be unknown as well. In this case, by changing the definition of $N$ to $N=-g(x) D+$ $g(x) \Delta u$ and considering $\|\Delta u\| \leq \xi(t)$ such that $\xi(t)$ is a known positive function, the results obtained so far will be valid by modifying $\dot{z}, \widehat{N}$, and $\tau$ as bellow.

$\dot{z}=-K \sigma+g \tau-\dot{v}_{d}-\left(\alpha_{0}(t)+\xi(t)\right) \lambda_{\max }\left(M^{-1}\right) \operatorname{sgn}(\sigma)-\varepsilon \operatorname{sig}^{\vartheta}(\sigma)-|f(x, v)| \operatorname{sgn}(\sigma)$ $\widehat{N}=-K \sigma-f(x, v)-\varepsilon \operatorname{sig}^{\vartheta}(\sigma)-\left(\alpha_{0}+\xi\right) \lambda_{\max }\left(M^{-1}\right) \operatorname{sgn}(\sigma)-|f(x, v)| \operatorname{sgn}(\sigma)$ 
$\tau=g^{-1}\left(-f(x, v)-\operatorname{sig}^{\chi_{x}}\left(e_{v}\right)-\operatorname{sig}^{\chi_{v}}\left(\phi\left(e_{x}, e_{v}\right)\right)+\dot{v}_{d}+\tau_{r}\right)$

Remark 5. The obtained results are applicable for actuators act as non-symmetric nonlinear dead-zone functions.

\section{Design of Full Order Nonlinear Finite Time Velocity Observer}

From now on it is assumed that velocity $v$ is not measurable directly however, according to Assumption 4, position $x$ is still available. The following nonlinear observer is designed to estimate position and velocity at an adjustable finite time. Since all state variables of robot manipulator (4) can be reconstruct by this observer, it is called the full order finite time nonlinear observer.

$\dot{\hat{x}}=\widehat{v}-\left(\varsigma_{0}+\varsigma_{3}\right) \operatorname{sig}(\widehat{x}-x)-h(x, \widehat{v}) \operatorname{sgn}(\widehat{x}-x)-\varsigma_{2} \operatorname{sig}^{\beta}(\widehat{x}-x)$

$\dot{\hat{v}}=-\varsigma_{1} \hat{v}-\varsigma_{3}|\widehat{x}-x|+f(x, \widehat{v})+g(x) u$

$h(x, \widehat{v})=\varsigma_{1} \kappa+\lambda_{\max }\left(M^{-1}\right) \alpha_{0}(t)+(\|\widehat{v}\|+\kappa) \theta_{2}(x, \widehat{v})+\varsigma_{2}(\|\widehat{v}\|+\kappa)^{\beta}+\theta_{1}(x, \widehat{v})$

Here $\hat{x}=\left[\begin{array}{lll}\hat{x}_{1} & \cdots & \hat{x}_{n}\end{array}\right]^{T}$ and $\hat{v}=\left[\begin{array}{lll}\hat{v}_{1} & \cdots & \hat{v}_{n}\end{array}\right]^{T}$ are estimates of $x$ and $v$, respectively. Positive constants $\varsigma_{0}, \varsigma_{2}, \varsigma_{3}$, and $0<\beta<1$ are chosen by designer and $\theta_{1}(x, \hat{v})=$ $2 \lambda_{\max }\left(M^{-1}\right)(\|B\|+\|C\|)\|\hat{v}\|^{2}, \theta_{2}(x, \hat{v})=\lambda_{\max }\left(M^{-1}\right)(\|B\|+\|C\|)(\|\hat{v}\|+\kappa)$, and $\varsigma_{1}=\left(1+\varsigma_{0}\right)$. To facilitate the proof of the finite time convergence of $\hat{x}$ and $\hat{v}$ to $x$ and $v$, Proposition 2 is presented.

Proposition 2. $f(x, v) \in \mathbb{R}^{n}$, defined in (6), fulfills the following inequality.

$\|f(x, \hat{v})-f(x, v)\| \leq \theta_{1}(x, \hat{v})+\theta_{2}(x, \hat{v})\|\hat{v}-v\|$.

Proof. According to definition of $f(x, v)$ in $(6)$, it is concluded that

$\|f(x, \hat{v})-f(x, v)\| \leq\left\|M^{-1} B(\langle v, v\rangle-\langle\hat{v}, \hat{v}\rangle)\right\|+\left\|M^{-1} C\left(v^{2}-\hat{v}^{2}\right)\right\|$

By referring to properties of 2-norm of vectors, one concludes that 
$\|f(x, \hat{v})-f(x, v)\| \leq \lambda_{\max }\left(M^{-1}\right)\left(\|B\|(\|\langle v, v\rangle\|+\|\langle\hat{v}, \hat{v}\rangle\|)+\|C\|\left(\left\|v^{2}\right\|+\left\|\hat{v}^{2}\right\|\right)\right)$

By considering definitions of $\langle v, v\rangle$ and $v^{2}$, inequalities $\|\langle v, v\rangle\| \leq\|v\|^{2}$ and $\left\|\hat{v}^{2}\right\| \leq\|v\|^{2}$ are concluded (proofs are given in the Appendices). Using these inequalities, (36) is simplified to

$\|f(x, \hat{v})-f(x, v)\| \leq \lambda_{\max }\left(M^{-1}\right)\left(\|B\|\left(\|v\|^{2}+\|\hat{v}\|^{2}\right)+\|C\|\left(\|v\|^{2}+\|\hat{v}\|^{2}\right)\right)$.

This inequality is reformulated as follow.

$$
\begin{gathered}
\|f(x, \hat{v})-f(x, v)\| \leq \lambda_{\max }\left(M^{-1}\right)\left(2(\|B\|+\|C\|)\|\hat{v}\|^{2}+(\|B\|+\|C\|)\left(\|v\|^{2}-\|\hat{v}\|^{2}\right)\right) \\
\leq \lambda_{\max }\left(M^{-1}\right)\left(2(\|B\|+\|C\|)\|\hat{v}\|^{2}+(\|B\|+\|C\|)(\|v\|+\|\hat{v}\|)(\|v\|-\|\hat{v}\|)\right)
\end{gathered}
$$

Considering Assumption 3, the following inequality is obtained.

$\|f(x, \hat{v})-f(x, v)\| \leq \lambda_{\max }\left(M^{-1}\right)\left(2(\|B\|+\|C\|)\|\hat{v}\|^{2}+(\|B\|+\|C\|)(\kappa+\|\hat{v}\|)\|\hat{v}-v\|\right)$

By considering definitions of positive functions $\theta_{1}(x, \hat{v})$ and $\theta_{2}(x, \hat{v})$, inequality (34) is concluded. This completes the poof.

Using Theorem 4, $\hat{x}$ and $\hat{v}$ are proven to reach $x$ and $v$ in a tunable finite time $T_{S_{o}}$ by applying observer (33). Assumptions 5 and 6 are considered on $u$ and manipulator (4) to be used in proof of Theorem 4 .

Assumption $5[30,31] . u \in \mathbb{R}^{n}$ belongs to the extended space $\ell_{p e}^{n}$ with $1 \leq p<\infty$. That is, any truncation of $u$, described by

$u_{\mathfrak{J}}(t)=\left\{\begin{array}{cll}u(t) & \text { if } & 0 \leq t<I \\ 0 & \text { if } & \mathfrak{J} \leq t\end{array}\right.$

fulfills inequality $\sqrt[p]{\int_{0}^{\infty}\left\|u_{\mathfrak{I}}(t)\right\|^{p} d t}<\infty$ where $\mathfrak{I}$ is a constant.

Since $u$ is output of the saturation function, this assumption is reasonable.

Assumption 6 [30, 31]. Robotic manipulator (4) might be unstable but it does not 
have a finite escape time. This means that states of the manipulator will not diverge to infinity in the small finite time.

Theorem 4. Consider robotic manipulator (4) that satisfies Assumptions 1, 3, 4, and 6 with input $u \in \mathbb{R}^{n}$ that satisfies Assumption 5. Then, by using observer (33), estimates $\hat{x}$ and $\hat{v}$ exactly converge to $x$ and $v$ in finite time $T_{S_{o}}$ bounded as

$T_{S_{o}} \leq\left(\varsigma_{0}(1-\beta)\right)^{-1}\left(\ln \left(\varsigma_{0}\left(\left\|\tilde{e}_{x}(0)\right\|+\left\|\tilde{e}_{v}(0)\right\|\right)^{1-\beta}+\varsigma_{2}\right)-\ln \varsigma_{2}\right)$,

where $\tilde{e}_{x}=\hat{x}-x$ and $\tilde{e}_{v}=\hat{v}-v$ are the estimation errors

Proof. Based on Assumptions 1, 3, 5, and 6, estimation errors $\tilde{e}_{x}$ and $\tilde{e}_{v}$ could not have a finite escape time. Dynamics of the estimation errors are determined as below

$\dot{\tilde{e}}_{x}=\tilde{e}_{v}-\left(\varsigma_{0}+\varsigma_{3}\right) \operatorname{sig}\left(\tilde{e}_{x}\right)-h(x, \hat{v}) \operatorname{sgn}\left(\tilde{e}_{x}\right)-\varsigma_{2} \operatorname{sig}^{\beta}\left(\tilde{e}_{x}\right)$

$\dot{\tilde{e}}_{v}=-\varsigma_{1} \hat{v}-\varsigma_{3}\left|\tilde{e}_{x}\right|+f(x, \hat{v})-f(x, v)-N(t, x, v)$

Consider the candidate Lyapunov function as $V=\left\|\tilde{e}_{x}\right\|+\left\|\tilde{e}_{v}\right\|$. The time derivative of this function is $\dot{V}=\left\|\tilde{e}_{x}\right\|^{-1} \tilde{e}_{x}^{T} \dot{\tilde{e}}_{x}+\left\|\tilde{e}_{v}\right\|^{-1} \tilde{e}_{v}^{T} \dot{\tilde{e}}_{v}$. By defining $\left(\tilde{e}_{x}\right)_{j}$ as the $j^{\text {th }}$ component of vector $\tilde{e}_{x}, \sum_{j=1}^{n}\left|\left(\tilde{e}_{x}\right)_{j}\right| \geq\left\|\tilde{e}_{x}\right\|$ and $\sum_{j=1}^{n}\left|\left(\tilde{e}_{x}\right)_{j}\right|^{\beta+1} \geq\left\|\tilde{e}_{x}\right\|^{\beta+1}$ are deduced from inequalities (i) and (ii) of Lemma 2. By placing (42) into $\dot{V}$ and using the mentioned inequalities, one obtains the following relation

$$
\begin{array}{r}
\dot{V} \leq\left\|\tilde{e}_{x}\right\|^{-1} \tilde{e}_{x}^{T} \tilde{e}_{v}-\left(\varsigma_{0}+\varsigma_{3}\right)\left\|\tilde{e}_{x}\right\|-h(x, \widehat{v})-\varsigma_{2}\left\|\tilde{e}_{x}\right\|^{\beta}-\varsigma_{1}\left\|\tilde{e}_{v}\right\|-\varsigma_{1}\left\|\tilde{e}_{v}\right\|^{-1} \tilde{e}_{v}^{T} v \\
-\varsigma_{3}\left\|\tilde{e}_{v}\right\|^{-1} \tilde{e}_{v}^{T}\left|\tilde{e}_{x}\right|+\left\|\tilde{e}_{v}\right\|^{-1} \tilde{e}_{v}^{T}(f(x, \widehat{v})-f(x, v))-\left\|\tilde{e}_{v}\right\|^{-1} \tilde{e}_{v}^{T} N
\end{array}
$$

By employing Cauchy-Schwartz inequalities $\left|\tilde{e}_{x}^{T} \tilde{e}_{v}\right| \leq\left\|\tilde{e}_{x}\right\|\left\|\tilde{e}_{v}\right\|,\left|\tilde{e}_{v}^{T} v\right| \leq\left\|\tilde{e}_{v}\right\|\|v\|$, $\left|\tilde{e}_{v}^{T}\right| \tilde{e}_{x}\|\leq\| \tilde{e}_{v}\|\|\left|\tilde{e}_{x}\right|\left\|\quad, \quad\left|\tilde{e}_{v}^{T} N\right| \leq\right\| \tilde{e}_{v}\|\| N \| \quad, \quad$ and $\quad\left|\tilde{e}_{v}^{T}(f(x, \hat{v})-f(x, v))\right| \leq\left\|\tilde{e}_{v}^{T}\right\|$ $\|f(x, \hat{v})-f(x, v)\|$, the following inequality is obtained.

$\dot{V} \leq\left\|\tilde{e}_{v}\right\|-\left(\varsigma_{0}+\varsigma_{3}\right)\left\|\tilde{e}_{x}\right\|-h(x, \hat{v})-\varsigma_{2}\left\|\tilde{e}_{x}\right\|^{\beta}-\varsigma_{1}\left\|\tilde{e}_{v}\right\|+\varsigma_{1}\|v\|+\varsigma_{3}\left\|\tilde{e}_{x}\right\|+$ 
$\|f(x, \hat{v})-f(x, v)\|+\|N\|$

By replacing for $h(x, \hat{v})$ and using Proposition 2, inequality $\|v\| \leq \kappa$ from Assumption 3 , inequality $\|N\| \leq \lambda_{\max }\left(M^{-1}\right) \alpha_{0}(t)$, and definition $\varsigma_{1}=\left(1+\varsigma_{0}\right)$, this relation is simplified to

$\dot{V} \leq-\varsigma_{0}\left\|\tilde{e}_{x}\right\|-\varsigma_{0}\left\|\tilde{e}_{v}\right\|-\varsigma_{2}\left\|\tilde{e}_{x}\right\|^{\beta}-\varsigma_{2}(\|\hat{v}\|+\kappa)^{\beta}-(\|\hat{v}\|+\kappa) \theta_{2}+\left\|\tilde{e}_{v}\right\| \theta_{2}$

By applying $\left\|\tilde{e}_{v}\right\| \leq(\|\hat{v}\|+\kappa)$ and $-(\|\hat{v}\|+\kappa)^{\beta} \leq-\left\|\tilde{e}_{v}\right\|^{\beta}$, it concludes that

$\dot{V} \leq-\varsigma_{0}\left(\left\|\tilde{e}_{x}\right\|+\varsigma_{0}\left\|\tilde{e}_{v}\right\|\right)-\varsigma_{2}\left(\left\|\tilde{e}_{x}\right\|^{\beta}+\left\|\tilde{e}_{v}\right\|^{\beta}\right)$

Based on inequality $(i)$ of Lemma 2 and considering definition $V=\left\|\tilde{e}_{x}\right\|+\left\|\tilde{e}_{v}\right\|$, inequality $-\left(\left\|\tilde{e}_{x}\right\|^{\beta}+\left\|\tilde{e}_{v}\right\|^{\beta}\right)<-\left(\left\|\tilde{e}_{x}\right\|+\left\|\tilde{e}_{v}\right\|\right)^{\beta}=-V^{\beta}$ is obtained. By utilizing this inequality, (46) can be written as $\dot{V}+\varsigma_{0} V+\varsigma_{2} V^{\beta} \leq 0$. By setting $\rho_{1}=\varsigma_{0}, \rho_{2}=$ $\varsigma_{2}$, and using Lemma 1 , it is concluded that there is a finite time $T_{S_{o}}$, introduced by (41), such that $\tilde{e}_{x}=0$ and $\tilde{e}_{v}=0$ for $t \geq T_{S_{o}}$. This means that estimates $\hat{x}$ and $\hat{v}$ converge to $x$ and $v$ for times larger than $T_{S_{o}}$. This completes the proof.

Remark 6. The proposed observer in (33) and settling time $T_{S_{o}}$ depend on arbitrary positive coefficients $\varsigma_{0}, \varsigma_{2}, \varsigma_{3}$, and $0<\beta<1$ that with reasonable choice could increase the convergence rate of the estimations.

Referring to the assumption that velocity $v$ is not measurable and it is estimated by observer (33), the proposed torque in (10) and disturbance observer (8) should be modified with replacing of $v$ by its estimation $\hat{v}$. The modified input torque is suggested as follows to ensure that the desired finite time tracking is achieved for closed-loop manipulator (4) in the tunable finite settling time $T_{s}^{\prime}=T_{s_{o}}+T_{S_{r}}+T_{S_{s}}$. Theorem 5 is given to prove this claim. 
$\bar{\tau}_{m}=\left\{\begin{array}{cll}{ }^{o} \tau & \text { for } \quad 0 \leq t<T_{S_{o}} \\ \tau \text { is defined by (8) }-(10) & \text { for } & T_{s_{o}} \leq t\end{array}\right.$

where ${ }^{o} \tau \in \mathbb{R}^{n}$ is an arbitrary absolutely continuous function and $v$ is replaced by $\hat{v}$ for all $t \geq 0$ in (8)-(10) with initial conditions $\tau\left(T_{S_{o}}\right)={ }^{o} \tau\left(T_{S_{o}}\right)$.

Theorem 5. Consider that $x$ is the only measurable state in manipulator (4) and $v$ is estimated by finite time observer (33). Then, control input (47) ensures that joints' positions of closed loop manipulator (4) reach the desired trajectories $x_{d} \in \mathbb{R}^{n}$ within a finite settling time $T_{s}^{\prime}$ less or equal to $T_{S_{o}}+T_{S_{r}}+T_{S_{S}}$ where $T_{S_{o}}$ satisfies inequality (41) and settling times $T_{S_{r}}$ and $T_{S_{S}}$ fulfill the following inequalities.

$T_{s_{r}} \leq(\eta(1-\vartheta))^{-1}\left(\ln \left(\eta \sqrt{\left(\sum_{j=1}^{n}\left(s_{j}^{2}\left(t=T_{s_{o}}\right)+\sigma_{j}^{2}\left(t=T_{s_{o}}\right)\right)\right)^{1-\vartheta}}+\gamma\right)-\ln \gamma\right)$

$T_{S_{s}} \leq \max _{j}\left\{\frac{\left(3-\chi_{x_{j}}\right)}{\left(c_{j}\left(1-\chi_{x_{j}}\right)\right)}\left(V_{j}\left(e_{x_{j}}\left(t=T_{S_{o}}+T_{s_{r}}\right), e_{v_{j}}\left(t=T_{S_{o}}+T_{s_{r}}\right)\right)\right)^{\frac{1-\chi x_{j}}{3-\chi x_{j}}}\right\}$

Proof. For $0 \leq t \leq T_{s_{o}}$, an arbitrary input torque ${ }^{o} \tau \in \mathbb{R}^{n}$ is applied to joints of manipulator (4) and its state $x$ is supplied as input to observer (33) that estimates $v$. According to Assumptions 5 and 6, velocity estimate $\hat{v}$, as output of observer (33), does not diverge to infinity for interval $0 \leq t \leq T_{S_{o}}$. Referring to Theorem 4 , it is concluded that for $t \geq T_{S_{o}}$, $\hat{v}$ becomes equal to $v$ and, in consequence, modified input torque $\bar{\tau}_{m}$, describing by (47), becomes equal to $\tau$ defined by (10). Therefore, by referring to Theorems 1 and 3 , it is found that $x$ exactly converges to $x_{d}$ in an adjustable finite time $T_{s}^{\prime}=T_{S_{o}}+T_{S_{r}}+T_{S_{S}}$ where $T_{s_{o}}$ is determined by (41). It is obvious that in inequalities (11) and (23), related to the settling times $T_{S_{r}}$ and $T_{S_{S}}$, initial conditions at times $t=0$ and $t=T_{S_{r}}$ should be replaced by ones at times 
$t=T_{s_{o}}$ and $t=T_{s_{o}}+T_{s_{r}}$. By these replacements, inequalities (48) are obtained. This completes the proof.

The proposed torque in (47) has been constructed based on measurability of position $x$ as the available output, velocity estimation $\hat{v}$ as the output of observer (33), and disturbance estimation $\widehat{N}$ as the output of disturbance observer (8). A schematic block diagram of the proposed output feedback control of the closed-loop manipulator is shown in Fig. 1.

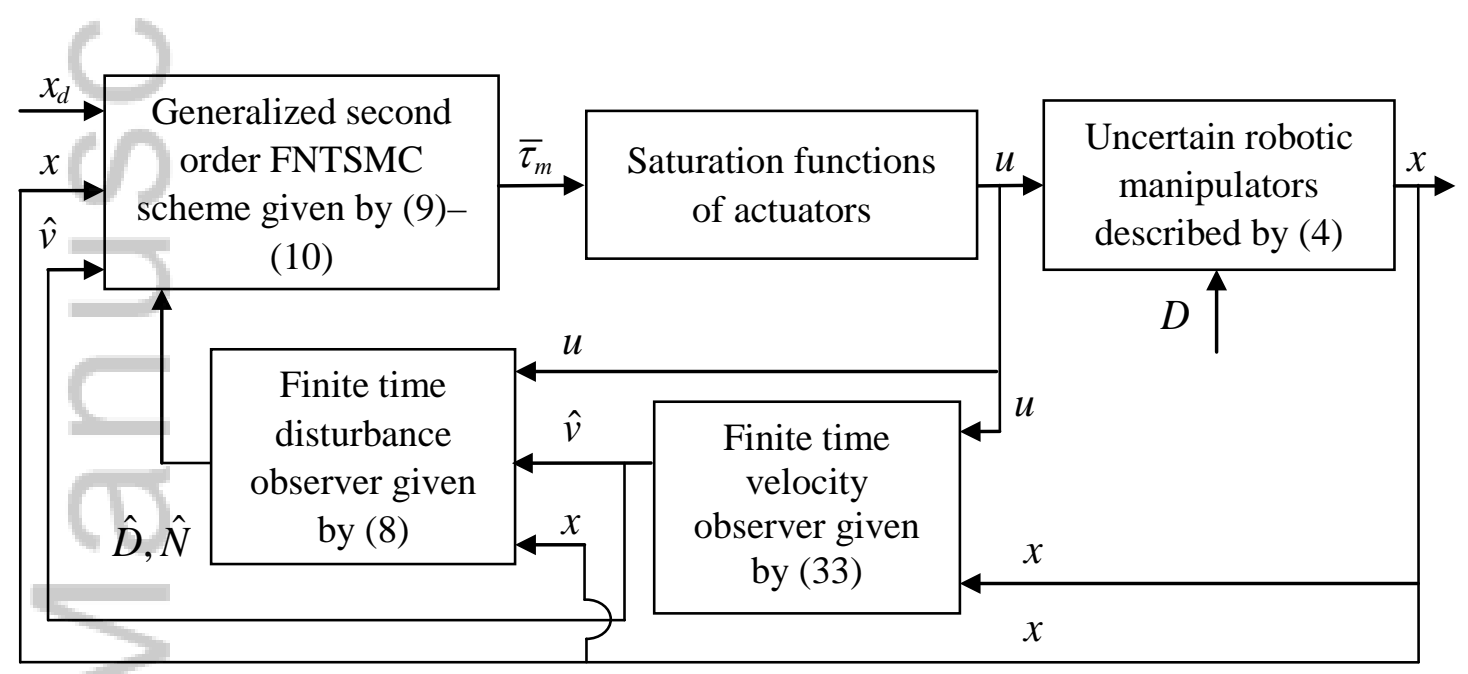

Fig. 1. A schematic of the FNTSMC-based output feedback control of manipulator.

Table 1 illustrates all optional parameters of the suggested control scheme with their required conditions. In order to tune these parameters, one can consider a cost function including two main terms related to the energy of control torques and finite time convergence rate $\left(T_{S_{o}}, T_{S_{r}}\right.$, and $\left.T_{S_{S}}\right)$. Based on this cost function and solving an optimization problem, proper values of the parameters could be determined. In addition, intelligent methods such as genetic algorithm and ant colony could be applied for tuning these parameters. 
Table 1. Introducing the optional parameters and their required conditions

\begin{tabular}{|l|l|l|}
\hline Controllers and Observers & Parameters & Requirements \\
\hline Sliding Mode Controller & $\chi_{x_{j}}, \omega_{j}, \Omega_{j}$ & $0<\chi_{x_{j}}<1, \omega_{j}>0, \Omega_{j}>0$ \\
\hline Velocity Observer & $\varsigma_{0}, \varsigma_{2}, \varsigma_{3}, \beta$ & $\varsigma_{0}>0, \varsigma_{2}>0, \varsigma_{3}>0,0<\beta<1$ \\
\hline Disturbance Observer & $k_{j}, \varepsilon_{j}, \vartheta$ & $k_{j}>0, \varepsilon_{j}>0,0<\vartheta<1$ \\
\hline
\end{tabular}

\section{Numerical Simulation}

In this part, the obtained results are simulated on a 2-link robotic manipulator where its dynamics fulfill (4) [36]. Matrices $M, B, C$, and $G$ of this manipulator with $n=2$ are given as

$M=\left[\begin{array}{cc}7.96+2.4 \cos \left(x_{2}\right) & 0.96+1.2 \cos \left(x_{2}\right) \\ 0.96+1.2 \cos \left(x_{2}\right) & 5.96\end{array}\right], B=\left[\begin{array}{c}-2.4 \sin \left(x_{2}\right) \\ 0\end{array}\right]$,

$C=\left[\begin{array}{cc}-1.2 \sin \left(x_{2}\right) & 0 \\ 0 & 1.2 \sin \left(x_{2}\right)\end{array}\right], G=\left[\begin{array}{c}19.6 \cos \left(x_{1}\right)+11.76 \cos \left(x_{1}+x_{2}\right) \\ 11.76 \cos \left(x_{1}+x_{2}\right)\end{array}\right]$.

The initial conditions of the manipulator are considered as $x(0)=\left[\begin{array}{ll}-\pi / 3 & -4 \pi / 3\end{array}\right]^{T}$ and $v(0)=\left[\begin{array}{ll}0 & 0\end{array}\right]^{T}$. The desired trajectory is selected as $x_{d}(t)=[\sin (8 t) \cos (8 t)]^{T}$. Initial values for observer (33) are chosen as $\hat{x}(0)=\left[\begin{array}{ll}0 & 0\end{array}\right]^{T}$ and $\hat{v}(0)=\left[\begin{array}{ll}5 & -6\end{array}\right]^{T}$. The disturbance is considered as $D=\left[\begin{array}{lll}d_{1}(t) & d_{2}(t)\end{array}\right]^{T}=0.1 x(t)+(0.5+$ $0.5 \sin (t))\left[\begin{array}{ll}1 & 1\end{array}\right]^{T}$. Based on Assumption 2, for the considered saturation functions $u_{1}$ and $u_{2}, u_{\max _{j}}$ and $u_{\min _{j}}, j=1,2$ are assumed as $u_{\max _{1}}=u_{\max _{2}}=60$ and $u_{\min _{1}}=u_{\min _{2}}=40$. According to Assumption 3, the constant parameter $\kappa$ is selected as $\kappa=25$. The design coefficients of observer (33) and controller (47) are chosen as follows. Also, the arbitrary input ${ }^{o} \tau$ in (47) is selected as $\tau$ given by (10).

$\varepsilon_{1}=\varepsilon_{2}=20, k_{1}=k_{2}=10, \vartheta=0.1, \chi_{1}=0.5, \chi_{3}=0.5, \omega_{1}=\omega_{2}=5, \Omega_{1}=$ $\Omega_{2}=3, \beta=0.9, \varsigma_{0}=\varsigma_{2}=\varsigma_{3}=1.5$. 


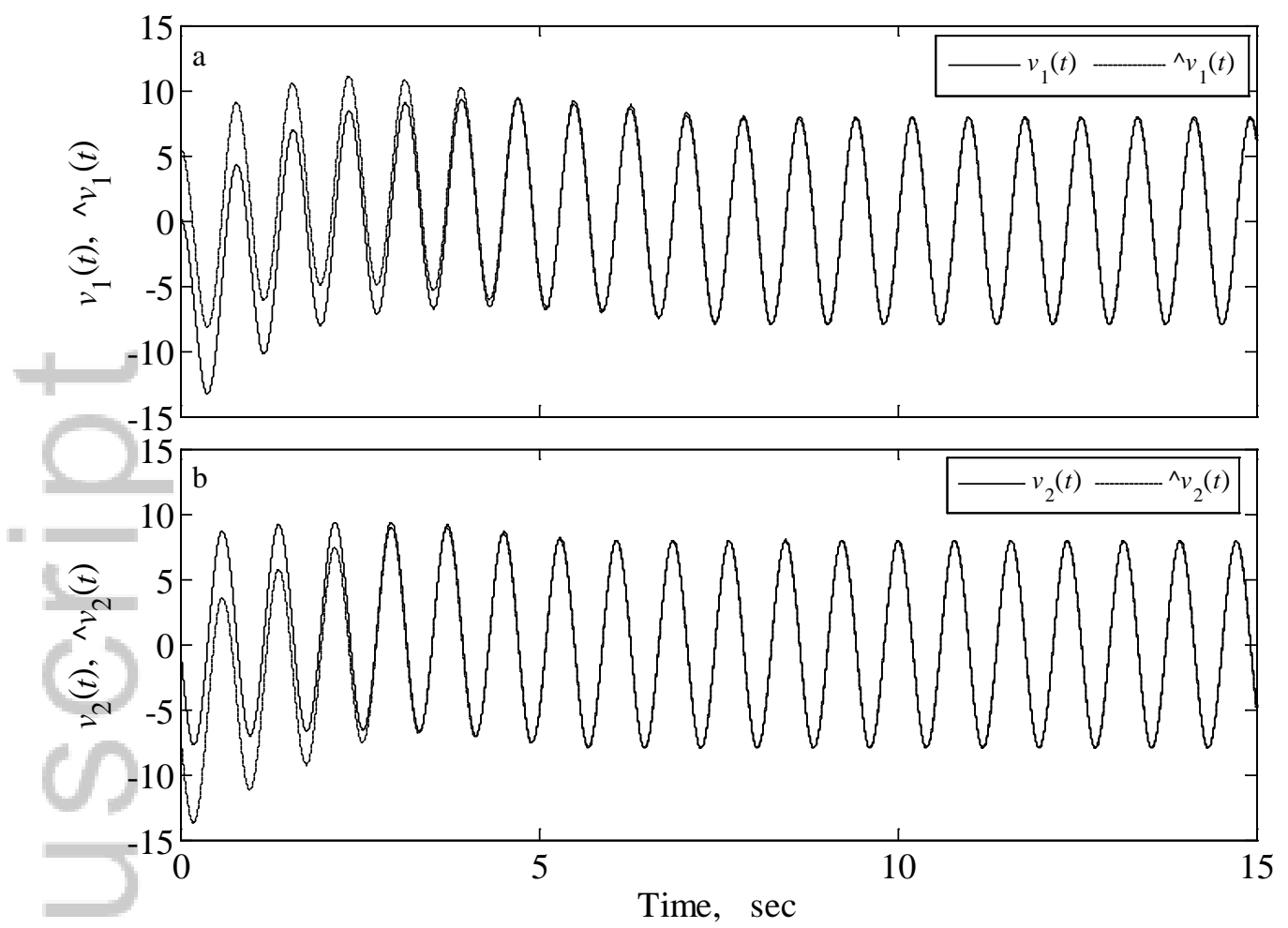

Fig. 2. a: Time responses of $\hat{v}_{1}(t)$ and $v_{1}(t)$, b: Time responses of $\hat{v}_{2}(t)$ and $v_{2}(t)$.

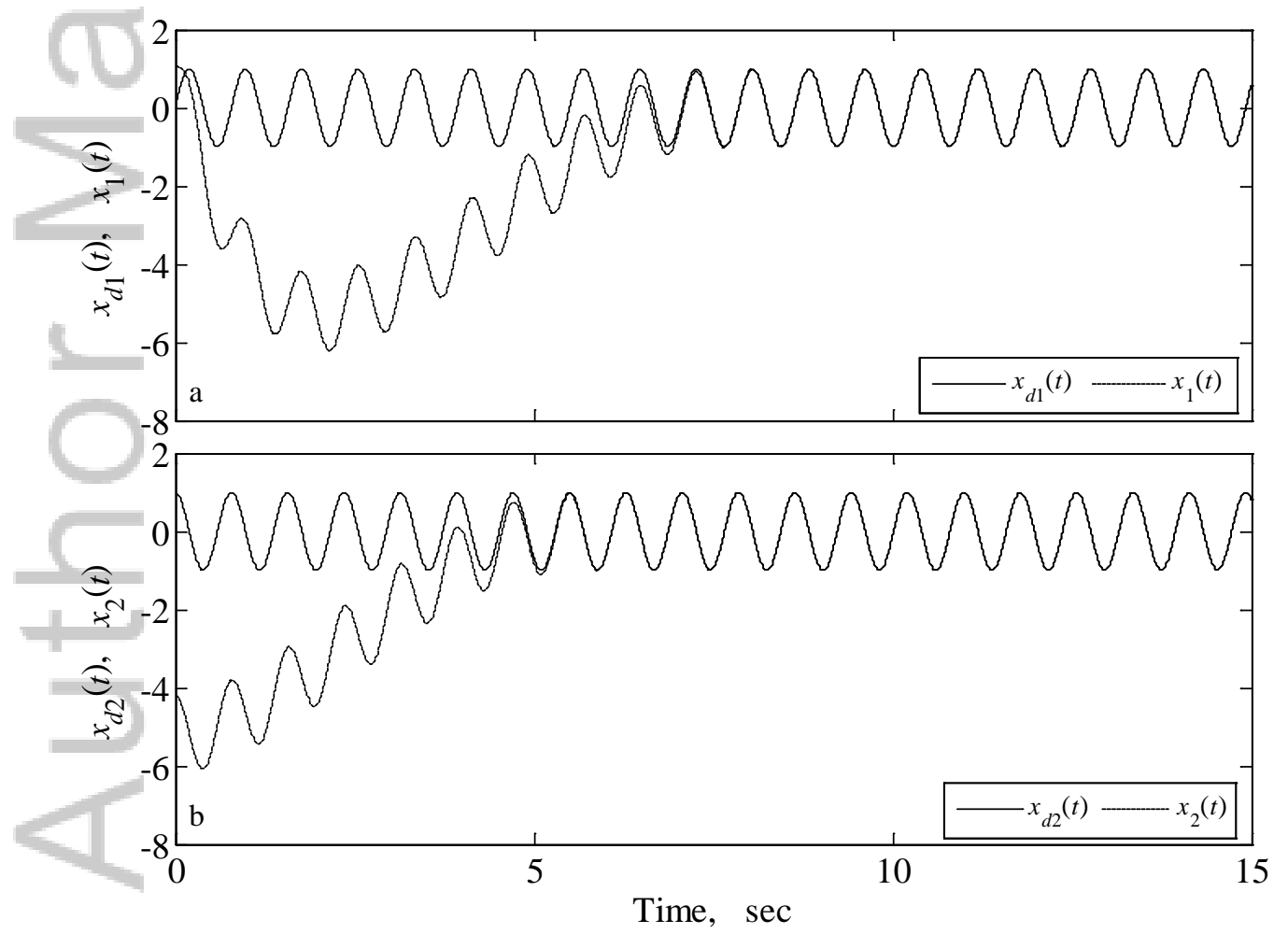

This article is protected by copyright. All rights reserved. 
Fig. 3. a: Time responses of $x_{1}(t)$ and $x_{d_{1}}(t)$, b: Time responses of $x_{2}(t)$ and $x_{d_{2}}(t)$. Results of numerical simulation are shown in Figs. 2-5. Figures $2 \mathrm{a}$ and $2 \mathrm{~b}$ show that the estimated velocities $\hat{v}_{1}(t)$ and $\hat{v}_{2}(t)$ reach actual velocities $v_{1}(t)$ and $v_{2}(t)$ within $T_{s_{o}} \approx 4(\mathrm{sec})$. Figures $3 \mathrm{a}$ and $3 \mathrm{~b}$ depict positions $x_{1}(t)$ and $x_{2}(t)$ of manipulator (4) that track selected desired trajectories $x_{d_{1}}(t)=\sin (8 t)$ and $x_{d_{2}}(t)=\cos (8 t)$, respectively, in the total finite settling time $T_{s}^{\prime} \approx 8(\mathrm{sec})$. Figures $4 \mathrm{a}$ and $4 \mathrm{~b}$ display input torques $\bar{\tau}_{m_{1}}$ and $\bar{\tau}_{m_{2}}$ where they are inputs for actuators connected to two joints of manipulator (4). From Fig. 4, it can be seen that chattering does not exist in the control laws. Finally, Figs. 5a and 5b illustrate estimated disturbances $\hat{d}_{1}(t)$ and $\hat{d}_{2}(t)$ that reach actual values $d_{1}(t)$ and $d_{2}(t)$.

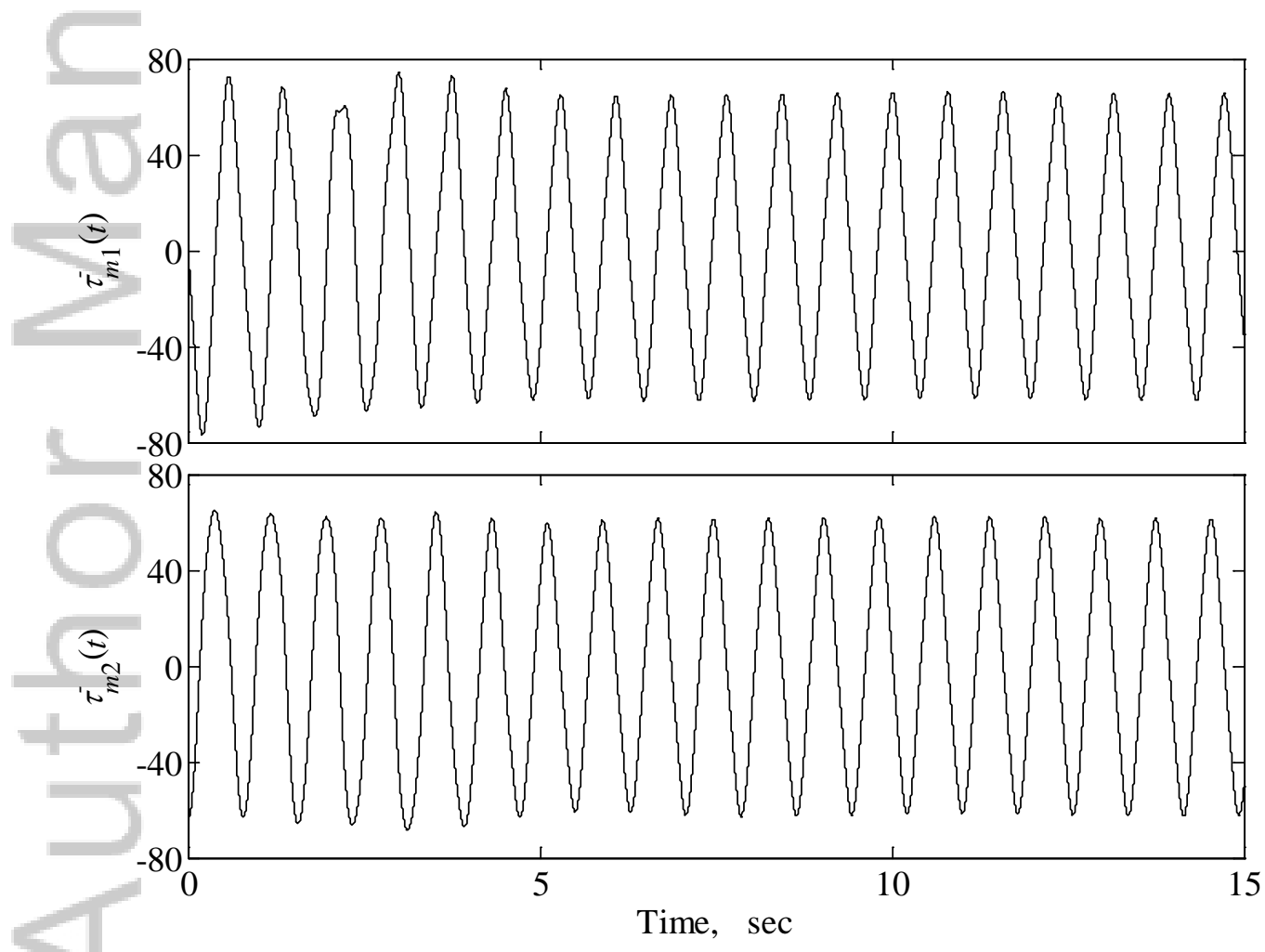

Fig. 4. Time responses of $\bar{\tau}_{m_{1}}$ and $\bar{\tau}_{m_{2}}$. 


\section{Conclusions}

The finite time tracking problem for robotic manipulators considering limitations of actuators and disturbances has been addressed where only positions of joints are measurable states. The proposed control algorithm has been created by combining three major components including a finite time velocity observer, a finite time disturbance observer, and a new class of modified second order FNTSMC. New terminal sliding surfaces and control laws were designed such that both reaching phase and sliding motion had finite time convergence property. Theoretical analysis proved that the outputs of disturbance and velocity observers converge precisely to their actual values within the finite times. Furthermore, the existence of a total finite settling time for converging of tracking errors to zero has been demonstrated. The presented control strategy, which does not need the manipulator's accelerations, has some advantages compared with other methods briefed at the end of introduction section. The provided numerical simulation example verified the analytical results presented in the paper.

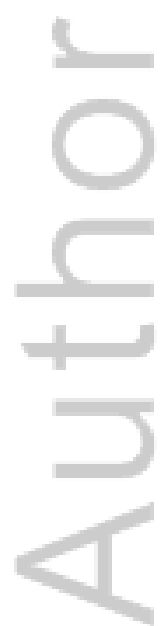

This article is protected by copyright. All rights reserved. 

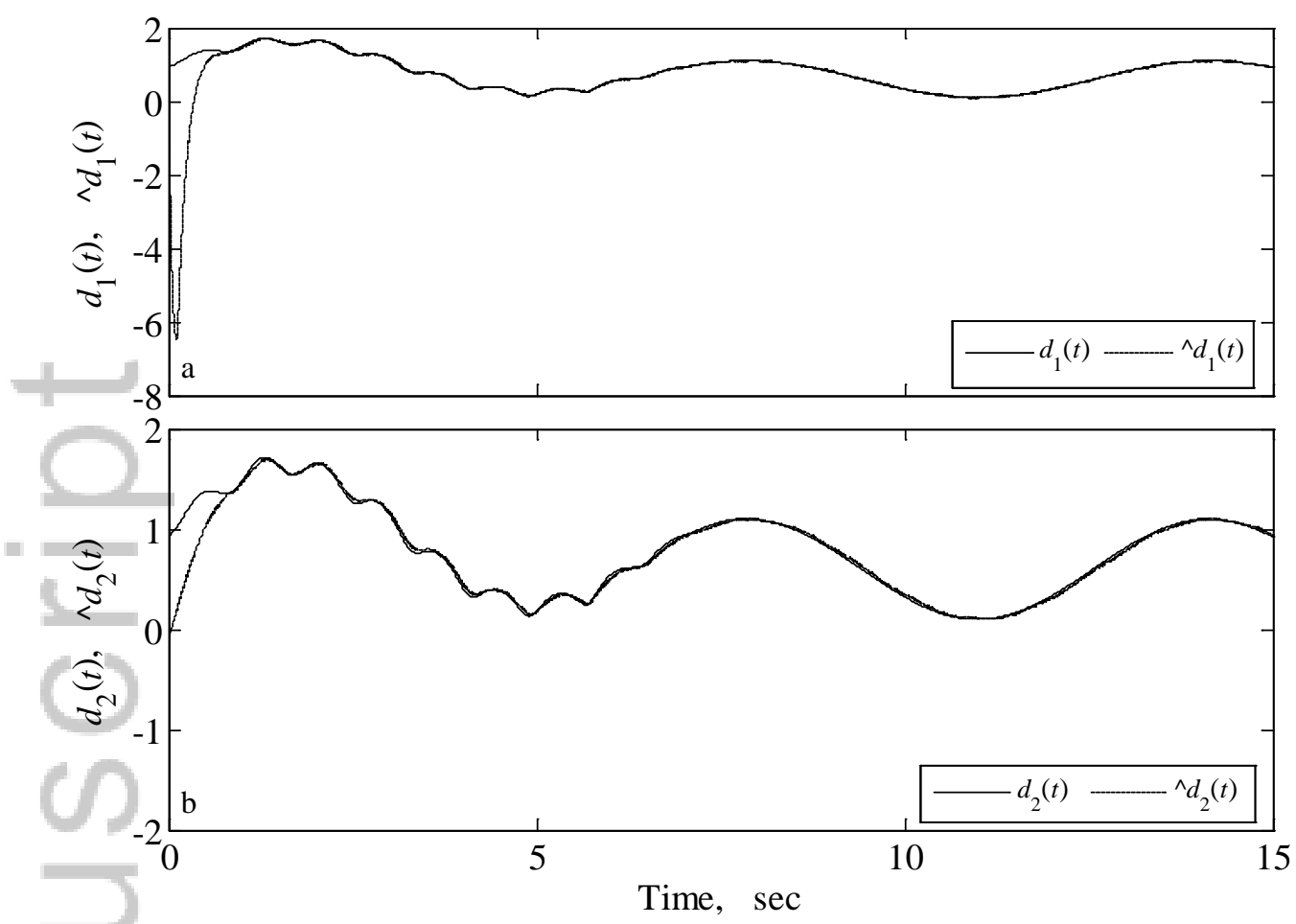

Fig 5. a: Time responses of $\hat{d}_{1}(t)$ and $d_{1}(t)$, b: Time responses of $\hat{d}_{2}(t)$ and $d_{2}(t)$.

\section{References}

[1] T.H.S. Li and Y.C. Huang, "MIMO adaptive fuzzy terminal sliding mode controller for robotic manipulators," Information Sciences, vol. 180, no. 23, pp. 4641-4660, 2010.

[2] H.G. Sage, M.F. De Mathelin, and E. Ostertag, "Robust control of robot manipulators: A survey," International Journal of Control, vol. 72, no. 16, pp. 1498-1522, 1999.

[3] C.C. Cheng, S.H. Chein, and F.C. Shih, "Design of robust adaptive variable structure tracking controllers with applications to rigid robot manipulators," IET Control Theory \& Applications, vol. 4, no. 9, pp. 1655-1664, 2010.

[4] A. Ferrara and L. Magnani, "Motion control of rigid robot manipulators via first 
and second order sliding modes," Journal of Intelligent and Robotic Systems, vol. 48, no. 1, pp. 23-36, 2007.

[5] S.H. Hsu and L.C. Fu, "A fully adaptive decentralized control of robot manipulators," Automatica, vol. 42, no. 10, pp. 1761-1767, 2006.

[6] V. Parra-Vega, S. Arimoto, Y.H. Liu, G. Hirzinger, and P. Akella, "Dynamic sliding PID control for tracking of robot manipulators: theory and experiments," IEEE Transactions on Robotics and Automation, vol. 19, no. 6, pp. 967-976, 2003.

[7] S.P. Bhat and D.S. Bernstein, "Continuous finite-time stabilization of the translational and rotational double integrators," IEEE Transactions on Automatic Control, vol. 43, no. 5, pp. 678-682, 1998.

[8] Y. Orlov, Y. Aoustin, and C. Chevallereau, "Finite time stabilization of a perturbed double integrator -part I: continuous sliding mode-based output feedback synthesis," IEEE Transaction on Automatic Control, vol. 56, no. 3, pp.614-618, 2011.

[9] Y. Su, "Global continuous finite-time tracking of robot manipulators," International Journal of Robust and Nonlinear Control, vol. 19, no. 17, pp. $1871-1885,2009$

[10] Y. Su and C. Zheng, "Global finite-time inverse tracking control of robot manipulators," Robotics and Computer-Intelligent Manufacturing, vol. 27, no. 3, pp. 550-557, 2011

[11] Y. Tang, "Terminal sliding mode control for rigid robots," Automatica, vol. 34, no. 1, pp.51-56, 1998. 
[12] Z. Man, A.P. Paplinski, and H. Wu, "A robust MIMO terminal sliding mode control scheme for rigid robotic manipulators," IEEE Transactions on Automatic Control, vol. 39, no. 12, pp. 2464-2469, 1994.

[13] D. Zhao, S. Li, and F. Gao, "A new terminal sliding mode control for robotic manipulators," International Journal of control, vol. 82, no. 10, pp. 1804-1813, 2009.

[14] Y. Wu, X. Yu, and Z. Man, "Terminal sliding mode design for uncertain dynamic systems," Systems \& Control Letters, vol. 34, no. 5, pp. 281-287, 1998.

[15] M. Chen, Q. X. Wu, and R. X. Cui, “Terminal sliding mode tracking control for a class of SISO uncertain nonlinear systems," ISA Transactions, vol. 52, no. 2, pp. 198-206, 2013.

[16] D. Zhao, S. Li, Q. Zhu, and F. Gao, "Robust finite time control approach for robotic manipulators," IET Control Theory \& Applications, vol. 4, no. 1, pp. 115,2010

[17] Y. Feng, X. Yu, and Z. Man, "Non-singular terminal sliding mode control of rigid manipulator," Automatica, vol. 38, no. 12, pp. 2159-2167, 2002.

[18] M. Jin, J. Lee, P.H. Chang, and C. Choi, "Practical nonsingular terminal sliding mode control of robot manipulators for high-accuracy tracking control," IEEE Transactions on Industrial Electronics, vol. 56, no. 9, pp. 3593-3601, 2009.

[19] M. Ghasemi, S. G. Nersesov, and G. Clayton, "Finite-time tracking using sliding mode control," Journal of the Franklin Institute, vol. 351, no. 5, pp. 2966-2990, 2014.

[20] G. Bartolini, E. Usai, A. Ferrara, and V.I. Utkin, “On multi-input chattering-free 
second-order sliding mode control," IEEE Transactions on Automatic Control, vol. 45, no. 9, pp. 1711-1717, 2000.

[21] A. Levant and A. Michael, “Adjustment of high-order sliding mode controllers," International Journal of Robust and Nonlinear Control, vol. 19, no. 15, pp. 1657-1672, 2009.

[22] S. Laghrouche, F. Plestan, and A. Glumineau, "Higher order sliding mode control based on integral sliding mode," Automatica, vol. 43, no. 3, pp. 531-537, 2007.

[23] M. Galicki, "Finite-time control of robotic manipulators," Automatica, vol. 51, no. 1, pp. 49-54, 2015.

[24] S. Mondal and C. Mahanta, "Adaptive second order terminal sliding mode controller for robotic manipulators," Journal of the Franklin Institute, vol. 351, no. 4, pp. 2356-2377, 2014.

[25] Q. Hu, B. Xiao, and P. Shi, "Tracking control of uncertain Euler-Lagrange systems with finite-time convergence," International Journal of Robust and Nonlinear Control, vol. 25, no. 17, pp. 3299-3315, 2015.

[26] H. Liu, T. Zhang, and X. Tian, "Continuous output-feedback finite -time control for a class of second-order nonlinear systems with disturbances," International Journal of Robust and Nonlinear Control, vol. 26, no. 2, pp. 218-234, 2016.

[27] C.P. Tan, X. Yu, and Z. Man, "Terminal sliding mode observers for a class of nonlinear systems," Automatica, vol. 46, no. 8, pp. 1401-1404, 2010.

[28] A. Gutierrez-Giles and M.A. Arteaga-Perez, "GPI based velocity/force observer design for robot manipulator," ISA Transactions, vol. 53, no. 4, pp. 929-938, 
2014.

[29] J. Hernandez and J.P. Barbot, "Sliding observer-based feedback control for flexible joints manipulator,” Automatica, vol. 32, no. 9, pp. 1243-1254, 1996.

[30] J.M. Daly and D.W.L. Wang, "Output feedback sliding mode control in the presence of unknown disturbances," Systems \& Control Letters, vol. 58, no. 3, pp. 188-193, 2009.

[31] D. Zhao, S. Li, and Q. Zhu, "Output feedback terminal sliding mode control for a class of second order nonlinear systems," Asian Journal of Control, vol. 15, no. 1, pp. 1-11, 2013.

[32] Z. Zhang and S. Xu, "Observer design for uncertain nonlinear systems with unmodeled dynamics," Automatica, vol. 51, no. 1, pp. 80-84, 2015.

[33] A. Laib, "Adaptive output regulation of robot manipulators under actuator constraints," IEEE Transactions on Robotics and Automation, vol. 16, no. 1, pp. 29-35, 2000.

[34] Y. Su and J. Swevers, "Finite-time tracking control for robot manipulators with actuator saturation," Robotics and Computer-Integrated Manufacturing, vol. 30, no. 2, pp. 91-98, 2014.

[35] A. Mohammadi, M. Tavakoli, H.J. Marquez, and F. Hashemzadeh, "Nonlinear disturbance observer design for robotic manipulators," Control Engineering Practice, vol. 21, no. 3, pp. 253-267, 2013.

[36] S. Yu, X. Yu, B. Shirinzadeh, and Z. Man, "Continuous finite-time for robotic manipulators with terminal sliding mode," Automatica, vol. 41, no. 11, pp.19571964, 2005.

This article is protected by copyright. All rights reserved. 
[37] Z. Zuo and L. Tie, “A new class of finite-time nonlinear consensus protocols for multi-agent systems," International Journal of Control, vol. 87, no. 2, pp.363370, 2014.

[38] G.H. Hardly, J.E. Littlewood, and G. Polya "Inequalities," Cambridge University Press, 1952.

Appendix A. Proof of inequality $\|\langle v, v\rangle\| \leq\|v\|^{2}$.

Based on definitions of vectors $\langle v, v\rangle \in \mathbb{R}^{n \times 0.5(n-1)}$ and $v \in \mathbb{R}^{n}$, it is apparent that

$\|\langle v, v\rangle\|^{2}=\frac{1}{2} \sum_{i=1}^{n} \sum_{j=1}^{n} x_{i}^{2} x_{j}^{2}$ and $\|v\|^{4}=\sum_{i=1}^{n} \sum_{j=1}^{n} x_{i}^{2} x_{j}^{2}+\sum_{i=1}^{n} x_{i}^{4}$.

Therefore, $\|\langle v, v\rangle\|^{2} \leq\|v\|^{4}$ and, in consequence, $\|\langle v, v\rangle\| \leq\|v\|^{2}$.

Appendix B. Proof of Inequality $\left\|v^{2}\right\| \leq\|v\|^{2}$.

According to definitions of vectors $v^{2} \in \mathbb{R}^{n}$ and $v \in \mathbb{R}^{n}$, it is clear that

$\left\|v^{2}\right\|=\left(\sum_{i=1}^{n} x_{i}^{4}\right)^{\frac{1}{2}}$ and $\|v\|^{2}=\sum_{i=1}^{n} x_{i}^{2}$.

By setting $\left|a_{i}\right|=x_{i}^{4}, \beta=0.5$, and applying inequality (ii) of Lemma 2 , it concludes that $\left(\sum_{i=1}^{n} x_{i}^{4}\right)^{\frac{1}{2}} \leq \sum_{i=1}^{n} x_{i}^{2}$ and, in consequence, $\left\|v^{2}\right\| \leq\|v\|^{2}$.

This article is protected by copyright. All rights reserved. 


\section{University Library}

\section{- M M I N E R VA A gateway to Melbourne's research publications}

Minerva Access is the Institutional Repository of The University of Melbourne

Author/s:

Abooee, A;Moravej Khorasani, M;Haeri, M

Title:

Finite time control of robotic manipulators with position output feedback

Date:

2017-11-10

Citation:

Abooee, A., Moravej Khorasani, M. \& Haeri, M. (2017). Finite time control of robotic manipulators with position output feedback. International Journal of Robust and Nonlinear Control, 27 (16), pp.2982-2999. https://doi.org/10.1002/rnc.3721.

Persistent Link:

http://hdl.handle.net/11343/292304 